INJE-TP-95-4

\title{
Hawking temperature from scattering off the charged 2D black hole
}

\author{
Jin Young Kim \\ Division of Basic Science, Dongseo University, Pusan 616-010, Korea \\ H. W. Lee and Y. S. Myung \\ Department of Physics, Inje University, Kimhae 621-749, Korea
}

\begin{abstract}
The charged 2D black hole is visualized as presenting an potential barrier $V^{\text {OUT }}\left(r^{*}\right)$ to on-coming tachyon wave. Since this takes the complicated form, an approximate form $V^{A P P}\left(r^{*}\right)$ is used for scattering analysis. We calculate the reflection and transmission coefficients for scattering of tachyon off the charged 2D black hole. The Hawking temperature is also derived from the reflection coefficient by Bogoliubov transformation. In the limit of $Q \rightarrow 0$, we recover the Hawking temperature of the 2D dilaton black hole.
\end{abstract}


Lower dimensional theories of gravity provide the simplified contexts in which to study black hole physics [1]. The non-triviality of these models arises from the non-minimal coupling of the dilaton to the scalar curvature. A dilaton potential of the type produced by the string loop corrections may induce multiple horizons [2]. For example, the event (outer) horizon $\left(r_{+}\right)$as well as inner (Cauchy) horizon $\left(r_{-}\right)$exist in the 2D charged black hole from heterotic string theories. In this sense the charged 2D black hole is very similiar to ReissnerNordström black hole. In the Reissner- Nordström geometry the stability of Cauchy horizon is one of the most interesting issues [3]. Recently we have shown that the Cauchy horizon is unstable because it is a surface where infalling tachyon got infinitely blueshifted [4].

In this paper, we study the scattering of tachyon exterior of charged 2D black hole. It is always possible to visualize the black hole as presenting an effective potential barrier (or well) to the on-coming waves. One easy way of understanding the attributes of a physical system (black hole) is to find out how it reacts to external perturbations. In the case of black hole, this is the very popular because there is no other way in which an external observer can explore the interior region of the horizon. One may expect that some of the incident wave will be irreversibly absorbed by the black hole, while the remaining fraction will be scattered back to infinity. There are two interesting quantum mechanical phenomena between the tachyon and black hole. One is the scattering of tachyonic mode off the black hole [5]. We calculate the reflection and transmission coefficients about this scattering. The other is the celebrated Hawking radiation [6]. In the case of charged black holes, the potential barrier $V^{\text {OUT }}\left(r^{*}\right)$ turns out to be a complicated form. Here the simplified form $V^{A P P}\left(r^{*}\right)$ is used for scattering analysis. We derive the Hawking temperature of the charged 2D black hole from the on-shell reflection coefficient by using the Bogoliubov transformation.

From the conformal invariance of the heterotic 2D string theories, one can derive the $\beta$-function equations $[2,7]$

$$
\begin{gathered}
R_{\mu \nu}+2 \nabla_{\mu} \nabla_{\nu} \Phi-F_{\mu \rho} F_{\nu}{ }^{\rho}-\frac{1}{2} \nabla_{\mu} T \nabla_{\nu} T=0, \\
\nabla^{2} \Phi-2(\nabla \Phi)^{2}+\frac{1}{2} \alpha^{2}+\frac{1}{4} F^{2}+\frac{1}{2} T^{2}=0,
\end{gathered}
$$




$$
\begin{gathered}
\nabla_{\mu} F^{\mu \nu}-2\left(\nabla_{\mu} \Phi\right) F^{\mu \nu}=0, \\
\nabla^{2} T-2 \nabla_{\mu} \Phi \nabla^{\mu} T+2 T=0,
\end{gathered}
$$

where $F_{\mu \nu}=\partial_{[\mu} A_{\nu]}$ is the Maxwell field. The above equations are also derived from the requirement that the fields must be an extremum of the low-energy string action $[4,8]$

$$
S_{l-e}=\int d^{2} x \sqrt{-G} e^{-2 \Phi}\left\{R+4(\nabla \Phi)^{2}+\alpha^{2}-\frac{1}{2} F^{2}-\frac{1}{2}(\nabla T)^{2}+T^{2}\right\} .
$$

After deriving the equations of motion, we take the transformation for convenience

$$
-2 \Phi \rightarrow \Phi, \quad T \rightarrow \sqrt{2} T, \quad-R \rightarrow R .
$$

Then the equations of motion become

$$
\begin{aligned}
& R_{\mu \nu}+\nabla_{\mu} \nabla_{\nu} \Phi+F_{\mu \rho} F_{\nu}{ }^{\rho}+\nabla_{\mu} T \nabla_{\nu} T=0, \\
& (\nabla \Phi)^{2}+\nabla^{2} \Phi-\frac{1}{2} F^{2}-2 T^{2}-8=0, \\
& \nabla_{\mu} F^{\mu \nu}+\left(\nabla_{\mu} \Phi\right) F^{\mu \nu}=0, \\
& \nabla^{2} T+\nabla \Phi \nabla T+2 T=0,
\end{aligned}
$$

where we set $\alpha^{2}=8$. The charged black hole solution to the above equations are given by

$$
\bar{\Phi}=2 \sqrt{2} r, \quad \bar{F}_{t r}=Q e^{-2 \sqrt{2} r}, \quad \bar{T}=0, \quad \bar{G}_{\mu \nu}=\left(\begin{array}{cc}
-f & 0 \\
0 & f^{-1}
\end{array}\right),
$$

with

$$
f=1-\frac{m}{\sqrt{2}} e^{-2 \sqrt{2} r}+\frac{Q^{2}}{8} e^{-4 \sqrt{2} r},
$$

where $m$ and $Q$ are the mass and charge of the black hole, respectively. For convenience, we take $m=\sqrt{2}$. From $f=0$, the double horizons $\left(r_{ \pm}\right)$are given by

$$
r_{ \pm}=\frac{1}{2 \sqrt{2}} \log \left[\frac{1 \pm \sqrt{1-\frac{Q^{2}}{2}}}{2}\right]
$$


where $r_{+}\left(r_{-}\right)$correspond to the event (Cauchy) horizons. To study the propagation of string fields, we introduce the perturbations around the background solution as [4]

$$
\begin{aligned}
& F_{t r}=\bar{F}_{t r}+\mathcal{F}_{t r}=\bar{F}_{t r}\left[1-\frac{\mathcal{F}(r, t)}{Q}\right], \\
& \Phi=\bar{\Phi}+\phi(r, t), \\
& G_{\mu \nu}=\bar{G}_{\mu \nu}+h_{\mu \nu}=\bar{G}_{\mu \nu}[1-h(r, t)], \\
& T=\bar{T}+\tilde{t} \equiv \exp \left(-\frac{\bar{\Phi}}{2}\right)[0+t(r, t)] .
\end{aligned}
$$

Here we choose the metric perturbation $\left(h_{\mu \nu}\right)$ in such a way that the background symmetry should be restored in the perturbation level. This is important in studying all black holes $[9,10]$. One has to linearize $(7)-(10)$ in order to obtain the equations governing the perturbation. Before we proceed, we first check whether the graviton $(h)$, dilaton $(\phi)$ and Maxwell mode $(\mathcal{F})$ are physically propagating modes in the $2 \mathrm{D}$ charged black hole background. We consider the conventional counting of degrees of freedom. The number of degrees of freedom for the gravitational field $\left(h_{\mu \nu}\right)$ in $d$-dimensions is $(1 / 2) d(d-3)$. For $d=4$ Schwarzschild black hole, we obtain two degrees of freedom. These correspond to Regge-Wheeler mode for odd-parity perturbation and Zerilli mode for even-parity perturbation. We have -1 for $d=2$. This means that in two dimensions the contribution of graviton is equal and opposite to that of a spinless particle (dilaton). The graviton-dilaton modes are gauge degrees of freedom and thus cannot appear in the physical observables [9]. In addition, the Maxwell field has $d-2$ physical degrees of freedom. For $d=2$, Maxwell field has no physical degrees of freedom. Thus we insist that graviton-dilaton, and Maxwell modes are gauge artefacts in the charged 2D black hole. Since these are not physically propagating modes, it is not necessary to study eqs.(7)-(9). One remaining equation that describes a physically propagating mode is just the tachyon equation (10), which can be rewritten as

$$
f^{2} \partial_{r}^{2} t+2 \sqrt{2} f(1-f) \partial_{r} t-2 f(1-f) t-\partial_{t}^{2} t=0
$$

One way to study the tachyonic equation is to transform (18) into the usual one-dimensional Schrödinger equation by eliminating the linear derivative term. Introducing the coordinate 
transformation

$$
r \rightarrow r^{*} \equiv g(r)
$$

(18) can be rewritten as

$$
f^{2} g^{\prime 2} \frac{\partial^{2}}{\partial r^{* 2}} t+f\left\{f g^{\prime \prime}+f^{\prime} g^{\prime}\right\} \frac{\partial}{\partial r^{*}} t-\left[\sqrt{2} f f^{\prime}-2 f(1-f)\right] t-\frac{\partial^{2}}{\partial t^{2}} t=0
$$

where the prime (I) denotes the derivative with respect to $r$. Requiring that the coefficient of the linear derivative vanish, one finds the relation

$$
g^{\prime}=\frac{1}{f}
$$

Assuming $t\left(r^{*}, t\right) \sim t_{\omega}\left(r^{*}\right) e^{-i \omega t}$, one can cast (19) into one-dimensional Schrödinger equation

$$
\left\{\frac{d^{2}}{d r^{* 2}}+\omega^{2}-V_{T}(r)\right\} t_{\omega}=0
$$

where the effective potential $V(r)$ is given by

$$
V(r)=f\left(\sqrt{2} f^{\prime}-2(1-f)\right)
$$

When $Q=1, V(r)$ is a double-humped barrier well $\left(V^{I N}\right)$ between the Cauchy horizon and event horizon, while it is just a potential barrier $\left(V^{O U T}\right)$ outside the event horizon (see Fig.1). Here we are interested only in the scattering of tachyon by $V^{O U T}$ outside of the black hole. When $Q$ is small, this is approximated by the $Q=0$ case. This case corresponds to the $2 \mathrm{D}$ dilaton black hole background as

$$
\bar{\Phi}=2 \sqrt{2} r, \quad \bar{F}_{t r}=0, \quad \bar{T}=0, \quad \bar{G}_{\mu \nu}=\left(\begin{array}{cc}
-\tilde{f} & 0 \\
0 & \tilde{f}^{-1}
\end{array}\right)
$$

with

$$
\tilde{f}=1-e^{-2 \sqrt{2} r}
$$

The event horizon is shifted from $r_{+}=0$ (for $Q=0$ ) to $r_{+}=-0.056$ (for $Q=1$ ) and $r_{+}=-0.0004$ (for $Q=0.1$ ). However, this shift does not affect the scattering analysis outside the black hole. In this case we can introduce the explicit form of $r^{*}$ 


$$
r^{*} \equiv g(r)=r+\frac{1}{2 \sqrt{2}} \ln \left(1-e^{-2 \sqrt{2} r}\right)
$$

Note that $r^{*}$ ranges from $-\infty$ to $+\infty$, while $r$ ranges from the event horizon of the black hole $\left(r_{+}=0\right)$ to $+\infty$. Using this coordinate, one can rewrite the exterior potential $\left(V^{O U T}\right)$ in terms of $r^{*}$ as

$$
V^{\text {OUT }}\left(r^{*}\right)=\frac{1}{2\left(\cosh \sqrt{2} r^{*}\right)^{2}}\left[1-\frac{Q^{2}\left(6-2 \exp \left(-2 \sqrt{2} r^{*}\right)\right)}{16\left(1+\exp \left(2 \sqrt{2} r^{*}\right)\right)}-\frac{3 Q^{4} \exp \left(-2 \sqrt{2} r^{*}\right)}{64\left(1+\exp \left(2 \sqrt{2} r^{*}\right)\right)^{2}}\right] .
$$

Since the form of this potential is complicated, we will simplify the potential for scattering analysis. By noting that the second and last terms are small compared with the first, one approximate $V^{O U T}\left(r^{*}\right)$ as

$$
V^{A P P}\left(r^{*}\right)=\frac{1}{2\left(\cosh \sqrt{2} r^{*}\right)^{2}}\left[1-\delta^{2}(Q)\right]
$$

with $\delta^{2}(Q)=\left(\sqrt{\frac{4}{32}} Q\right)^{2}$. When $Q=0.1$, we have $V^{O U T}\left(r^{*}\right)=V^{A P P}\left(r^{*}\right)$ for the whole regions (see Fig.2). Even for $Q=1$, we find that $V^{O U T}\left(r^{*}\right)=V^{A P P}\left(r^{*}\right)$ is a good approximation on the right branch (see Fig.3). Therefore, when the energy $\left(\omega^{2}\right)$ is large and $Q \sim 1$ or charge $Q$ is small $(<1)$, we expect that there is not much difference in scattering analysis if we use $V^{A P P}\left(r^{*}\right)$ instead of $V^{O U T}\left(r^{*}\right)$.

Let us thus study with the equation

$$
\left\{\frac{d^{2}}{d r^{* 2}}+\omega^{2}-V^{A P P}\left(r^{*}\right)\right\} t_{\omega}\left(r^{*}\right)=0
$$

Following Ref. [11], the above is exactly solvable and $t_{\omega}\left(r^{*}\right)$ is given by

$$
\begin{aligned}
t_{\omega}\left(r^{*}\right) & =C_{1}\left(\cosh \sqrt{2} r^{*}\right)^{-2 \lambda} F\left(-\lambda+\frac{i \omega}{2 \sqrt{2}},-\lambda-\frac{i \omega}{2 \sqrt{2}}, \frac{1}{2} ; z\right) \\
& +C_{2}\left(\cosh \sqrt{2} r^{*}\right)^{-2 \lambda} \sqrt{z} F\left(-\lambda+\frac{1}{2}+\frac{i \omega}{2 \sqrt{2}},-\lambda+\frac{1}{2}-\frac{i \omega}{2 \sqrt{2}}, \frac{3}{2} ; z\right),
\end{aligned}
$$

where $z=-\left(\sinh \sqrt{2} r^{*}\right)^{2}$ and $\lambda=(\sqrt{4 / 32} Q-1) / 4 . C_{1}, C_{2}$ are two arbitrary constants and can be determined by imposing the appropriate boundary conditions. The boundary conditions are as follows. Asyptotically the solution consists of both ingoing $\left(\exp \left(-i \omega r^{*}\right): \longleftarrow\right)$ and outgoing $\left(R \exp \left(i \omega r^{*}\right): \longrightarrow\right)$, but at the horizon it is purely ingoing $\left(T \exp \left(-i \omega r^{*}\right): \longleftarrow\right)$. 
We call this type of solutions as $\left\{t_{\text {out }}\right\}$ and the corresponding vacuum state is defined as $|0\rangle_{\text {out }}$. The transmission amplitude $T$ (coefficient $\mathcal{T}=|T|^{2}$ ) and reflection amplitude $R$ (coefficient $\mathcal{R}=|R|^{2}$ ) are given by

$$
\begin{aligned}
T & =\left(\frac{1}{4}\right)^{-i \omega / \sqrt{2}} \exp (i \Delta) \frac{\sinh (\pi \omega / \sqrt{2})}{\sinh (\pi \omega / \sqrt{2})+i \cosh (\pi \delta(Q) / 2)}, \\
\mathcal{T} & =\frac{(\sinh (\pi \omega / \sqrt{2}))^{2}}{(\cosh (\pi \delta(Q) / 2))^{2}+(\sinh (\pi \omega / \sqrt{2}))^{2}}, \\
R & =\left(\frac{1}{4}\right)^{-i \omega / \sqrt{2}} \exp (i \Delta) \frac{1}{\cosh (\pi \delta(Q) / 2)-i \sinh (\pi \omega / \sqrt{2})}, \\
\mathcal{R} & =\frac{1}{(\cosh (\pi \delta(Q) / 2))^{2}+(\sinh (\pi \omega / \sqrt{2}))^{2}}
\end{aligned}
$$

where the phase factor $(\exp (i \Delta))$ is given by

$$
\exp (i \Delta)=\frac{\Gamma(i \omega / \sqrt{2})(\Gamma(1 / 4-i \omega / 2 \sqrt{2}))^{2}}{\Gamma(-i \omega / \sqrt{2})(\Gamma(1 / 4+i \omega / 2 \sqrt{2}))^{2}} .
$$

As might be expected, one finds that $\mathcal{T}+\mathcal{R}=1$.

Let us consider two limiting cases, $\omega \rightarrow 0$ and $\infty$, for future references. The first case reduces to $\mathcal{T}(\omega \rightarrow 0)=0$ and $\mathcal{R}(\omega \rightarrow 0)=1$. This corresponds to the total reflection since the potential becomes effectively an infinite potential barrier. The second case is given by

$$
\mathcal{T}(\omega \rightarrow \infty) \approx 1, \quad \mathcal{R}(\omega \rightarrow \infty) \approx \exp (-\sqrt{2} \pi \omega) \equiv \exp \left(-\frac{\omega}{T_{s t}}\right) .
$$

For this case the energy $\omega$ of the mode is much larger than the height $\left(\left(1-\delta^{2}(Q)\right) / 2\right)$ of the potential barrier. This corresponds to the classical picture of Boltzmann distribution with the statistical temperature $T_{s t}=\frac{1}{\sqrt{2} \pi}$.

In order to obtain the Hawking temperature, let us introduce another boundary conditions. Asymptotically the wave is purely ingoing $(\longleftarrow)$, but near the event horizon it has both outgoing $(\longrightarrow)$ and ingoing parts $(\longleftarrow)$. We denote this type of solutions as $\left\{t_{i n}\right\}$ and this vacumm state is defined as $|0\rangle_{\text {in }}$. The vacuum states $|0\rangle_{\text {out }}$ and $|0\rangle_{\text {in }}$ form two different bases of which any state can be expanded. Consequently these are two distinct Fock space vacuum states. The two sets $\left\{t_{\text {out }}\right\}$ and $\left\{t_{\text {in }}\right\}$ are related by the Bogoliubov transformation $[12,13]$. It is then a standard calculation to evaluate the expectaion value of the number opeartor $N^{\text {out }}$ in the vacuum state $|0\rangle_{\text {in }}$ 


$$
{ }_{i n}\left\langle 0\left|N^{\text {out }}\right| 0\right\rangle_{\text {in }}=\frac{1}{\left|\frac{C_{2}}{C_{1}}\right|^{2}-1}=\frac{\mathcal{R}}{1-\mathcal{R}},
$$

where $\mathcal{R}$ is the reflection coefficient for tachyon off the black hole in (33). Finally we define the Hawking temperature as

$$
{ }_{\text {in }}\left\langle 0\left|N^{\text {out }}\right| 0\right\rangle_{\text {in }}=\frac{1}{\exp \left(\frac{\omega}{T_{H}^{Q}}\right)-1} .
$$

From (35) and (36), we read off the Hawking temperature as

$$
T_{H}^{Q}=\frac{\omega}{\ln \left[1+\left(\frac{\sinh (\pi \omega / \sqrt{2})}{\cosh (\pi \delta(Q) / 2)}\right)^{2}\right]} .
$$

We obtain in the limit of $\delta \rightarrow 0(Q \rightarrow 0)$,

$$
T_{H}^{Q \rightarrow 0}=\frac{\omega}{2 \ln [\cosh (\pi \omega / \sqrt{2})]}
$$

which is just the Hawking temperature of $2 \mathrm{D}$ dilaton black hole [14]. In the limit of $Q \rightarrow 0$ and $\omega \rightarrow \infty, T_{H}^{Q}$ reduces to the statistical temperature $T_{s t}=\frac{1}{\sqrt{2} \pi}$ in (34). We see that for the out observers the in vacuum is full of particles in a heat bath at the temperature $T_{H}^{Q}$. Notice that this situation is static (eternal black hole): although the black hole emits the thermal radiation $(\longrightarrow)$, it does not lose its mass $m=\sqrt{2}$. This is because it absorbs an equal amount of matter $(\longleftarrow)$ from the heat bath outside the horizon [5].

In conclusion, the charged 2D black hole is visualized as presenting an potential barrier $V^{\text {OUT }}\left(r^{*}\right)$ to on-coming tachyon wave in the exterior region of black hole. Since this has a complicated form, we approximate this into $V^{A P P}\left(r^{*}\right)$. This is in a good agreement with $V^{\text {OUT }}\left(r^{*}\right)$ for either higher energy $\omega^{2}$ or small $Q$. We use $V^{A P P}\left(r^{*}\right)$ instead $V^{\text {OUT }}\left(r^{*}\right)$ for scattering analysis. In this case one-dimensional Schrödinger equation is exactly solvable. We calculate the reflection and transmission coefficients for scattering of tachyon off the charged 2D black hole. The Hawking temperature is also derived from the reflection coefficient by Bogoliubov transformation. In the limit of $Q \rightarrow 0$, we recover the Hawking temperature of the 2D dilaton black hole. 


\section{ACKNOWLEDGMENTS}

This work was supported in part by the Basic Science Research Institute Program, Min-

istry of Education, 1995, Project NO. BSRI-95-2413 and by NONDIRECTED RESEARCH FUND, Korea Research Foundation, 1994. 


\section{REFERENCES}

[1] E. Witten, Phys. Rev. D44 (1991) 314; G. Mandal, A. Sengupta and S. R. Wadia, Mod. Phys. Lett. A6 (1991) 1685; S.Elitzur, A. Forge and E. Rabinovici, Nucl. Phys. B 359 (1991) 581; S. P. deAlwis and J. Lykken, Phys. Lett. B269 (1991) 464.

[2] M. D. McGuigan, C. N. Nappi and S. A. Yost, Nucl. Phys. B 375 (1992) 421; O. Lechtenfeld and C. N. Nappi, Phys. Lett. B288 (1992) 72.

[3] R. Penrose, in Batelle Rencontres, edited by C. M. De Witt and J. A. Wheeler (Benjamin, New York, 1968); J. M. McNarama, Proc. Roy. Soc. Lon. A358 (1978) 499; Y. Gürsel, I. D. Novikov, V. D. Sandberg and A. A. Starobinsky, Phys. Rev. D19 (1979) 413; R. A. Matzer, N. Zamorano and V. D. Sandberg, Phys. Rev. D19 (1979) 2821; S. Chandrasekhar and J. Hartle, Proc. Roy. Soc. Lon. A384 (1982) 301.

[4] H. W. Lee, Y. S. Myung and J. Y. Kim, to be published.

[5] R. Dijkgraaf, H. Verlinde and E. Verlinde, Nucl. Phys. B371 (1992) 269.

[6] S. W. Hawking, Commun. Math. Phys. 43 (1975) 199.

[7] C.G. Callan, D. Friedan, E,J. Martinec, and M.J. Perry, Nucl. Phys. B262 (1985) 593; T. Banks, Nucl. Phys. B361 (1991) 166.

[8] A. Peet, L. Susskind and L. Thorlacius, Phys. Rev. D48 (1993) 2415.

[9] J.Y. Kim, H. W. Lee and Y. S. Myung, Phys. Lett. B328 (1994) 291; Y. S. Myung, Phys. Lett. B334 (1994) 29.

[10] S. Chandrasekhar, in Space and Geometry, edited by R. A. Matzner and L. C. Shepley (Texas Univ. Press, Austin, 1982).

[11] J.Y. Kim, H. W. Lee and Y. S. Myung, Phys. Rev. D50 (1994) 3942.

[12] K. Sfetsos, Nucl. Phys. B389 (1993) 424. 
[13] K. Ghoroku and A. L. Larsen, Phys. Lett. B328 (1994) 28.

[14] Y. S. Myung, J. Y. Kim and C. Jue, Phys. Lett. B341 (1995) 273. 


\section{FIGURES}

Fig.1: The $Q=1$ graph of the effective potential of tachyon $(V(r))$. This takes the double-humped barrier well $\left(V^{I N}\right)$ inside the black hole, while it takes a simple potential barrier $\left(V^{\text {OUT }}\right)$ outside the black hole. The event horizon is at $r_{+}=-0.056$ and the Cauchy horizon is at $r_{-}=-0.679$.

Fig.2 :The $Q=0.1$ graphs of potentials outside black hole. The solid (dashed) lines refer $V^{O U T}\left(r^{*}\right)\left(V^{A P P}\left(r^{*}\right)\right)$. In this case one finds that $V^{O U T}\left(r^{*}\right)=V^{A P P}\left(r^{*}\right)$ for whole regions.

Fig.3 :The $Q=1$ graphs of potentials outside black hole. The solid (dashed) lines refer $V^{\text {OUT }}\left(r^{*}\right)\left(V^{A P P}\left(r^{*}\right)\right)$. In this case one finds that $V^{O U T}\left(r^{*}\right) \sim V^{A P P}\left(r^{*}\right)$ for right branch. 